\title{
Obesity and muscle-macrophage crosstalk in humans and mice: A systematic review
}

Srikesh Rudrapatna ${ }^{1,2^{*}}$, Meha Bhatt ${ }^{1,2,3^{*}}$, Kuan-Wen Wang ${ }^{1,2}$, Rachel Bierbrier ${ }^{1,2,4}$, Pei-Wen Wang $^{1,2}$, Laura Banfield ${ }^{5}$, Wagdi Elsheikh ${ }^{1,2,3}$, E. Danielle Sims ${ }^{1,2}$, Devin Peterson ${ }^{6}$, Lehana Thabane ${ }^{1,3,7,8,9}$, Mark A. Tarnopolsky ${ }^{1,10}$, Gregory R. Steinberg ${ }^{10}$, M. Constantine Samaan ${ }^{1,2,3}$

${ }^{1}$ Department of Pediatrics, McMaster University, Hamilton, Ontario, Canada

${ }^{2}$ Division of Pediatric Endocrinology, McMaster Children's Hospital, Hamilton, Ontario, Canada

${ }^{3}$ Department of Health Research Methods, Evidence and Impact, McMaster University, Hamilton, Ontario, Canada

${ }^{4}$ Michael G. DeGroote School of Medicine, McMaster University, Hamilton, Ontario, Canada

${ }^{5}$ Health Science Library, McMaster University, Hamilton, Ontario, Canada

${ }^{6}$ Division of Orthopedics, Department of Pediatric Surgery, McMaster University, Hamilton, Ontario, Canada

${ }^{7}$ Department of Anesthesia, McMaster University, Hamilton, Ontario, Canada

${ }^{8}$ Centre for Evaluation of Medicines, Hamilton, ON, Canada

${ }^{9}$ Biostatistics unit, St Joseph's Healthcare-Hamilton, Hamilton, Ontario, Canada

${ }^{10}$ Department of Medicine, McMaster University, Hamilton, Ontario, Canada

*These authors contributed equally to this work

\section{Corresponding author:}

Dr. M. Constantine Samaan

Division of Pediatric Endocrinology,

Department of Pediatrics,

McMaster Children's Hospital,

McMaster University,

1280 Main Street West, HSC-3A57

Hamilton, Ontario L8S 4K1

Canada

Tel: 001-905-521-2100, ext. 75926

Fax: 001-905-308-7548

\section{E-mails of authors:}

Srikesh Rudrapatna: rudraps@mcmaster.ca

Meha Bhatt: meha.33@gmail.com

Kuan-Wen Wang: wangkww2@gmail.com

Rachel Bierbrier: rachel.bierbrier@medportal.ca

Pei-Wen Wang: cpw.wang7@gmail.com

Wagdi Elsheikh: Elsheikh@mcmaster.ca

E. Danielle Sims: simse@mcmaster.ca

This is the author manuscript accepted for publication and has undergone full peer review but has not been through the copyediting, typesetting, pagination and proofreading process, which may lead to differences between this version and the Version of Record. Please cite this article as doi: $10.1111 /$ obr.12922 
Laura Banfield: banfie@mcmaster.ca

Devin Peterson: dpeters@mcmaster.ca

Lehana Thabane: thabanl@mcmaster.ca

Mark Tarnopolsky: Tarnopol@mcmaster.ca

Gregory Steinberg: gsteinberg@mcmaster.ca

M. Constantine Samaan: samaanc@mcmaster.ca

Abstract

Obesity is associated with the production of inflammatory cytokines that are implicated in insulin resistance (IR), and if not addressed, can lead to type 2 diabetes (T2D). The role of the immune system in skeletal muscle (SM) inflammation and insulin sensitivity is not yet well characterized. As SM insulin resistance is an important determinant of glycemia, it is critical that the muscle-immune phenotype is mapped to design interventions to target T2D. This systematic review synthesized the evidence for SM macrophage content and phenotype in human and murine models of obesity, and the association of muscle macrophage content and phenotype with IR. Results were synthesized narratively, as we were unable to conduct a meta-analysis. We included 28 studies ( $\mathrm{n}=10$ human, $\mathrm{n}=18$ murine), and all studies detected macrophage markers in SM. Macrophage content was positively associated with IR. In humans and mice, there was variability in muscle macrophage content and phenotype in obesity. Overall certainty in the evidence was low due to heterogeneity in detection methods, and incompleteness of data reporting. Humans and mice show a presence of macrophage in SM in obesity and a positive association between macrophage content and IR, however there are differences in muscle macrophage phenotype in obesity. 


\section{INTRODUCTION}

One-in-three people around the world are affecting by overweight or obesity, and this is associated with an extensive global burden of noncommunicable diseases including myocardial infarction, stroke, hypertension, and type 2 diabetes (T2D) ${ }^{1-3}$. While the biopsychosocial drivers of obesity continue to be revealed ${ }^{4-7}$, one consistent phenomenon is the expansion of the adipose compartment $^{2,8}$. This expansion is associated with altered adipose tissue (AT) autocrine, paracrine, and endocrine functions with disrupted cellular metabolic signalling and insulin resistance (IR). If untreated, IR may progress to type 2 diabetes (T2D), a major contributor to cardiovascular disease risk $^{9-11}$.

While adipocyte expansion was considered as one of the main contributors to inflammation, contemporary insights have highlighted the role of immune cells in AT dysregulation and local inflammation and IR in obesity ${ }^{9,12-14}$. One of the most prominent cells in this immune-metabolic

crosstalk is the macrophage ${ }^{14}$. Macrophages are innate immune cells that exist on a spectrum of phenotypes that at its extremes include pro-inflammatory (M1) and anti-inflammatory (M2)

This article is protected by copyright. All rights reserved. 
macrophages ${ }^{15}$. In obesity, adipocyte production of chemokines including Chemokine C-C motif Ligand 2 (CCL2) and others creates a chemotactic gradient that attracts bone marrow-derived monocytes to exit the circulation and enter the AT and muscle. Sensing the local AT microenvironment, these cells differentiate to M1 macrophages, and there is a similar process of monocyte attraction evident in muscle ${ }^{1424}$. Excess adipose tissue M1 macrophage content has been linked, although inconsistently, to local tissue inflammation ${ }^{13,16,17}$. It is likely that intermediate macrophage phenotypes that harbor markers of both M1 and M2 cells are generated based on the local microenvironment, and these cells remain to be fully characterized ${ }^{18,19}$. As the AT is one of the main organs that expand in obesity, adipose tissue inflammation and adipocyte-macrophage crosstalk has been widely studied ${ }^{20-26}$. On the other hand, macrophage existence and actions in other metabolic organs including skeletal muscle (SM) has received less attention. There is a critical need to understand muscle-immune crosstalk in obesity, as SM accounts for the majority of postprandial glucose clearance, and its IR is a primary determinant of glucose dysmetabolism early in obesity ${ }^{27-29}$. Factors affecting SM insulin sensitivity, including inflammation, can contribute to increased risk for developing obesity-driven T2D, making it a potential target for intervention to mitigate the risk of diabetes ${ }^{1,30}$. Murine models have been used to provide critical insights into the pathogenesis of obesity ${ }^{31-36}$. Comparative transcriptional profiling suggests significant similarities in gene expression and their transcriptional regulation in murine and human monocytes but also some differences ${ }^{37}$. Whether murine muscle-immune crosstalk mimics that of humans in obesity is critical to clarify, so that 
therapeutic research findings are more confidently generalized from mice to humans. This will enhance the chances of success of moving obesity therapies from the bench to the clinic.

The aim of this systematic review is to summarize and assess the quality of the current evidence base for macrophage content and phenotype in SM in overweight/obesity in humans and murine models, and determine the association between muscle macrophage content and phenotype with IR.

\section{Objectives}

The primary objectives of this review are to evaluate the presence of macrophages in SM in humans and mice with overweight/obesity and assess their association with IR. The secondary objective is to assess muscle macrophage phenotype in humans and mice with overweight/obesity.

\section{METHODS}

This systematic review has been registered with PROSPERO (registration no.

CRD42016033035) and the methods are available in a published protocol ${ }^{38}$. This systematic review follows the Preferred Reporting Items for Systematic Reviews and Meta-Analyses (PRISMA) guidelines, and the completed PRISMA checklist is available in the supplementary materials (Table S1). We briefly report on the systematic review methods below.

\section{Data sources \& search strategy}

This article is protected by copyright. All rights reserved. 
We implemented a search strategy that collated evidence from several databases including MEDLINE, Embase, Web of Science, the Cochrane Library and SPORTDiscus from inception to December 2017. ProQuest Dissertations and Theses, the National Institutes of Health and the World Health Organization International clinical trials registries were searched as sources of grey literature. Additionally, we hand-searched reference lists of included studies. A sample of the search strategy was included in the published protocol for this review ${ }^{38}$.

\section{Eligibility criteria}

Multiple study designs were eligible for inclusion in this systematic review including cohort, cross-sectional and case-control studies that investigated macrophage infiltration into SM in humans and mice with overweight and obesity. We also aimed to include pilot and feasibility studies and conference abstracts and posters if applicable. We included studies reporting on the detection of macrophage markers in SM regardless of the technique used for detection.

Studies including patients with hypertension, chronic renal disease, autoimmune disease, cancer, pregnancy, smokers and athletes were excluded as these conditions are known to alter immune activation status. We also excluded studies with participants receiving parenteral nutrition, steroids, anti-inflammatory or immunomodulating therapies.

While we did set out initially to capture observational studies reporting macrophages in muscle in obesity, we did identify intervention-based studies for inclusion that reported pre-intervention data on macrophage markers, and also for a comprehensive picture of muscle-immune crosstalk in obesity and its modulation with interventions. 


\section{Outcome Measures}

The primary outcomes measured were macrophage content in SM in humans and mice with overweight/obesity and their association with IR. The secondary outcome was the phenotype of muscle-infiltrating macrophages in humans and mice with overweight/obesity.

\section{Study selection and data abstraction}

Two authors working independently within three teams reviewed the articles during each phase of the article selection process. For title and abstract selections, the reviewers screened the titles and abstracts emanating from the search results applying the inclusion and exclusion criteria to select eligible titles and abstracts. For the selected abstracts, the full-text papers were reviewed to determine suitability for inclusion in the systematic review. Disagreements were resolved to reach consensus through discussions among team members, and a third reviewer was consulted if consensus could not be reached.

\section{Quality of evidence assessment}

For human studies, we used the Newcastle Ottawa Scale (NOS) for case-control and cohort studies to assess the risk of bias ${ }^{39}$. We applied the Systematic Review Centre for Laboratory Animal Experimentation (SYRCLE) risk of bias tool to evaluate mice studies ${ }^{40}$. We also used the Grading of Recommendations, Assessment, Development and Evaluations (GRADE) framework tool to provide an overall assessment of the quality of evidence, as we noted that it can be applicable for the included studies, and this was not initially proposed in our protocol.

\section{Data synthesis}


Results from studies were synthesized narratively and reported in summary tables. Descriptive statistics were used to report the frequencies for measurements of macrophage content and the various macrophage markers present in SM. It was not possible to conduct a meta-analysis, as proposed in the a priori protocol due to the heterogeneity in experimental procedures and the lack of exact reported values. Furthermore, data were not comparable across studies, since presence of macrophage markers was reported as relative proportions within studies.

\section{RESULTS}

We screened 1090 unique citations and included data following the full text screening from 10 studies in adult human subjects ${ }^{41-50}$ and 18 studies in mice ${ }^{26,42,43,47,51-64}$. Figure 1 outlines the number of articles included at each phase in the screening process, including the reasons for exclusion. In reporting the data for humans and mice, we describe the muscle macrophage content first, followed by the macrophage phenotypes and their association with IR.

\section{Human studies}

The results from the human studies are summarized in Table 1. Ten studies were identified, including studies that reported interventions. Significant variation was noted in the reported study design, group comparisons, macrophage marker choice, method of macrophage marker detection, and statistical adjustments. There was a total of 228 participants in all studies included $^{41-50}$. The age range of participants was 20-61 years, and one study did not report on the age of participants ${ }^{48}$. Nine studies $(n=212,93 \%)$ reported on participants' sex distribution ${ }^{22}$, with the majority of participants being female $(n=148 ; 69.8 \%)$. 
Five studies compared adults who were lean (Body Mass Index (BMI) 18.5 to $<25 \mathrm{~kg} / \mathrm{m}^{2}$ ) to those with overweight $\left(\mathrm{BMI} \geq 25-29.9 \mathrm{~kg} / \mathrm{m}^{2}\right)$ and obesity $\left(\mathrm{BMI} \geq 30 \mathrm{~kg} / \mathrm{m}^{2}\right)^{41,43-45,47}$. There were 100 participants (43.9\%) with overweightedness or obesity across studies, and one study ( $\mathrm{n}=14)$ did not specify the number of subjects who were lean or with obesity ${ }^{48}$. While one study reported participants to be of normal weight, based on World Health Organization classification of BMI cutoffs of overweight and obesity in Asia ${ }^{65}$, many participants were in the overweight category, and we did end up including the study in our analysis ${ }^{50}$.

Of the 10 human studies included, heterogeneity was noted in study populations and comparison groups when it comes to body mass and glucose tolerance. Three studies reported on lean normal glucose tolerance (NGT) versus participants with obesity and NGT ${ }^{41,45,47}$, and two studies recruited three groups including patients who were lean and had NGT, patients with obesity and NGT, and those with overweight/obesity and $\mathrm{T} 2 \mathrm{D}^{43,44}$. One study included only patients with obesity, NGT and T2D ${ }^{42}$, two studies compared patients with NGT $(n=28)$ to those with impaired glucose tolerance (IGT, $\mathrm{n}=34)^{49,50}$, and the remaining two studies included lifestyle interventions included patients with obesity only ${ }^{46}$ and those with obesity and T2D ${ }^{48}(n=21,13$ female). These studies investigated $9-$ month $^{46}$ and 15 -week ${ }^{48}$ exercise programs that we ended up including in our analysis.

The molecular techniques used to detect macrophages and their phenotype included the assessment of mRNA abundance (Real-Time Polymerase Chain Reaction (RT-PCR) and Quantitative RT-PCR (qRT-PCR)), and protein detection (western blot, Enzyme-Linked 
Immunosorbent Assay (ELISA), and Immunohistochemistry (IHC)). One paper used

Transmission Electron Microscopy to determine macrophage content ${ }^{31}$. Different measures were used to report on insulin action and its association with macrophages. The use of Insulin Sensitivity Index (ISI), the frequently sampled intravenous glucose tolerance test (FSIVGTT), oral glucose tolerance test (OGTT), euglycemic hyperinsulinemic clamp, fasting plasma insulin, fasting plasma glucose, from which the Homeostatic Model Assessment-Insulin Resistance (HOMA-IR) and HOMA were calculated (Table 1).

\section{Muscle macrophage content in human studies}

All human studies identified macrophages in muscle ${ }^{41-50}$. While we had proposed in our protocol that we are going to perform subgroup analyses based on age and sex, the reported data formats did not allow such analyses. In addition, we had proposed certain markers to characterize macrophages in muscle however we summarized findings on the markers reported in the studies. The most common marker used to detect macrophages was the Cluster of Differentiation 68 $(\mathrm{CD} 68)^{66}$, a glycoprotein that is mainly localized to lysosomes and is expressed primarily in macrophages, and another marker used was CD40 (Table 1).

\section{Muscle macrophage phenotype in human studies}

Markers used to detect and broadly classify macrophage subtypes included those of inflammatory macrophages (CD14, CD16, CD11b, and Toll-Like Receptors (TLR) 2 and 4), anti-inflammatory or resident macrophages (CD14, CD16, CD163, CD206, CD11c the galactose-binding lectin (MGL) and Transforming Growth Factor $\beta 1$ (TGF- $\beta 1)$ ). The level of 
expression of some markers denotes whether the cell is an inflammatory or resident macrophage e.g. CD14 and CD16. In addition, the most important macrophage chemoattractant to muscle (Chemokine C-C motif Ligand 2 (CCL2)) was also measured. Several cytokines produced by the muscle cells themselves or by the immune cells within muscle were detected including mainly inflammatory cytokines (Tumor necrosis factor $\alpha$ (TNF $\alpha$ ), Interleukins (IL; IL-1 $\beta$, IL-6)) and anti-inflammatory cytokines (IL-10, IL-1 receptor antagonist a (IL-1Ra)) (Table 1).

\section{Association of muscle macrophages with obesity and glucose tolerance in human studies Comparison of lean versus obesity in subjects with NGT:}

In the three studies comparing individuals with NGT and lean status to those with NGT and obesity, there was conflicting evidence regarding the direction of the association between obesity and muscle macrophage content and variability in the markers used to ascertain muscle macrophage content and phenotype ${ }^{41,45,47}$. One study reported increased CD68 expression in subjects with obesity compared to those who were lean ${ }^{41}$, while another study reported significantly higher CD11b and a non-significant trend for higher CD11c in individuals with obesity compared to those who were lean, yet no difference in CCL2 expression, a major macrophage chemoattractant ${ }^{47}$. Macrophage content correlated negatively with insulin sensitivity $(\text { FSIVGTT })^{41}$. In contrast, one study reported lower macrophage content, reduced macrophage gene expression (CD68, CD11b, CD16, CD40, CD163, CD206,), with lower CCL2 and TNFa in SM of subjects with obesity compared to those who were lean, even after age and sex 
adjustments. IL- 6 and IL-10 were not detectable in most patients ${ }^{45}$. After controlling for BMI or the fat mass only CD40, CD206, and CD163 were positively correlated with ISI ${ }^{45}$.

Taken together, there is inconclusive evidence as to whether muscle macrophage markers change with obesity in NGT, and the exact phenotype of these macrophages is unclear. The increased macrophage marker expression is associated with IR.

\section{Comparison of subjects with NGT versus IGT:}

Between the two studies that compared subjects with NGT and IGT (without distinction of $\mathrm{BMI})^{49,50}$, there was conflicting evidence on the association of glucose tolerance with SM macrophage content. One study reported that CD68 and CCL2 mRNA is detectable in muscle at similar levels in subjects with NGT and IGT ${ }^{49}$. The other study found higher macrophage content on transmission electron microscopy and increased expression of CD68, TLR-4, TNF- $\alpha$ and IL-6 in subjects with IGT compared to those with NGT, and similar TLR-2 expression ${ }^{50}$. Therefore, there was similar or higher muscle macrophage content in sbjects with IGT compared to those with NGT, and cells had an inflammatory phenotype in IGT when a higher macrophage content was detected in muscle in the second study. There was no correlation between CD68 and insulin resistance.

\section{Comparison of subjects with NGT, lean and obesity versus subjects with type 2 diabetes} subjects:

In the two studies that compared subjects with NGT, lean/obesity to those with overweight/obesity and T2D, there was a trend for increased macrophages in $\mathrm{T}_{2} \mathrm{D}^{43}, 44$. One 
study found greater expression of macrophage markers CD11c and CD11b mRNA in subjects with obesity and T2D compared to those with obesity and NGT, yet this correlation was lost after adjustment of age. However CD206, CD163 and MGL mRNA abundance was similar between these two groups and TGF- $\beta 1$ mRNA abundance was lower in patients with obesity and T2D compared to those with obesity and $\mathrm{NGT}^{44}$. MGL and TGF- $\beta 1$ negatively correlated with HbA1c and fasting glucose levels. The other study reported greater CD68 and TNF- $\alpha$ mRNA abundance in subjects with obesity and T2D compared to those with NGT, lean and obesity, and CD68 correlated positively with HOMA-IR ${ }^{43}$. One study compared individuals with obesity and NGT and those with obesity and $\mathrm{T} 2 \mathrm{D}^{42}$, and found a non-significant trend of higher CD68 macrophages in subjects with T2D compared to those with NGT, and CD68 positively correlated with $\mathrm{BMI}^{42}$. CD68 and CD11c correlated positively with fasting plasma insulin and negatively with glucose disposal rate measured by hyperinsulinemic-euglycemic clamp, and CD68 also positively correlated with fasting plasma glucose $\mathrm{e}^{42}$.

Taken together, these data indicate that having T2D is associated with increased macrophage markers in muscle that is correlated positively with body mass. The exact phenotype of these macrophages is less clear, with some evidence of increased inflammatory and equal or reduced anti-inflammatory macrophage markers. Macrophage content was associated with insulin resistance in this population.

\section{Impact of interventions on muscle macrophage content and phenotype:}

Three studies investigated the effect of interventions on muscle macrophages ${ }^{46,48}$. 
In a nine-month exercise program in subjects with obesity and T2D, CD68 was detectable in muscle at baseline, with no change in macrophage content, HbA1c, or HOMA with the intervention $^{46}$.

In a 15-week lifestyle intervention in subjects with severe obesity, CD68, CD14, IL-8 and TNF $\alpha$ mRNA abundance were similar at baseline and post-intervention. However, IL-6 expression decreased significantly post-intervention ${ }^{48}$. Another study reported minimal changes in macrophage markers with exercise intervention ${ }^{26}$. In addition, there was no change in macrophage content when subjects with IGT were treated with the insulin sensitizer metformin and the anti-diabetic agent Pioglitazone ${ }^{49}$. These data suggest that current interventions do not alter macrophage content in muscle in obesity or diabetes.

\section{Risk of bias in human studies}

Table 2 presents a summary of the risk of bias assessment for human studies. Risk of bias was moderate to high for nine out of the ten studies ${ }^{41-49}$ and low for only one study ${ }^{50}$. Sources of bias included lack of description of participant recruitment strategies ${ }^{41-43,45-49}$ and unclear definition for the cases ${ }^{41,45-47}$ and controls ${ }^{41,42,46-49}$. Additionally, most studies did not conduct statistical analyses comparing cases and controls or adjust for confounders ${ }^{41-44,46-49}$.

\section{Murine studies}

The results from the 18 murine studies are presented in Table 3. Eight studies used C57BL/6 mice $^{43,51-56,59}$, nine used C57BL/6J mice ${ }^{26,42,47,57,58,60-63,67}$, one study used FVB/N mice ${ }^{64}$.

Studies also included other mouse models such as the ob/ob leptin deficient mice ${ }^{43,57}$ and the 
RCS10 mice with obesity ${ }^{53}$ In addition, IL-10 overexpression and CD11c knockout mice were also tested. All studies compared lean mice fed a standard chow diet (daily kcal from fat <20\%) to diet-induced mice with obesity fed a high fat diet (HFD, daily kcal from fat $>40 \%$ ). The age range of mice at the start of the dietary intervention was 4-12 weeks, and one study did not report the initial age of the mice ${ }^{43}$. The duration of high-fat feeding ranged from 2-24 weeks. The molecular techniques used to detect macrophages included gene expression (RT-PCR, qRT-PCR) and protein expression (western blot, ELISA, flow cytometry and IHC).

\section{Muscle macrophage content in murine studies}

All murine studies reported the presence of macrophages within skeletal muscle. The most frequent macrophage marker used was F4/80. In IHC analyses, macrophage markers were mostly localized to intermyocellular adipose depots $26,42,43,47,52,53,56,57,59$. and in one study they were detected between myofibers as well as in the intermyocellular fat compartment ${ }^{42}$. While most studies reported higher macrophage marker expression in mice with obesity compared to those who were lean $26,42,43,47,51-60,62,63,68$, some others did not report this association ${ }^{61,64}$. One study

reported lower mRNA abundance for IL-10 and Fractalkine receptor (CXC3CR1) ${ }^{51}$. Taken together, there data suggest that there is relatively consistent evidence for the increase in muscle macrophage marker expression in murine obesity.

\section{Muscle macrophage phenotype in murine studies}

Macrophage phenotyping used a broad set of macrophage markers. Pro-inflammatory (M1) macrophage markers used included CD11b, Toll-Like Receptors (TLR) 2 and 4, C-C chemokine 
receptor type 2 (CCR2), and Galectin-3 (MAC2). The anti-inflammatory (M2) macrophage markers included CD11c (a dendritic cell maker), CD206, CD163, CD301b, Fractalkine receptor (CX3CR1), Found in inflammatory zone 1 (Fizz1), and Macrophage galactose lectin 1 (MGL1). Other chemokines or their receptors were also tested (Table 2).

Out of eight studies that tested the gene expression of CD11 $c^{42,43,47,52,56,58,62,69}$, six reported elevated CD11c among mice with obesity compared to those who were lean ${ }^{42,43,47,52,56,69}$. Higher CD206 and CD301b and lower CX3CR1 expression was noted in mice with obesity compared to lean ones ${ }^{42,58,70}$. The evidence suggests that muscle macrophages in obesity trend towards an anti-inflammatory phenotype in mice.

\section{Association of muscle macrophages with obesity and glucose tolerance in murine studies}

Murine studies assessed glucose homeostasis and insulin action by measuring fasting blood glucose, glucose and insulin tolerance tests, HOMA-IR, 2-hour hyperinsulinemic-euglycemic clamp as well as protein kinase B (Akt) and insulin receptor substrate 1 (IRS-1) phosphorylations, which are markers of insulin signaling. These studies reported that mice with obesity had elevated fasting glucose and insulin levels, impaired glucose tolerance, and reduced insulin sensitivity compared to lean mice $43,47,53,57,59,62,64$.

Furthermore, four studies investigated the link between different muscle inflammation markers and $\mathrm{IR}^{43,53,57,59}$. The enhanced mRNA abundance of macrophage markers CD68 and CD11c negatively correlated with glucose tolerance measured via glucose tolerance tests (GTT) and insulin tolerance tests (ITT) ${ }^{24}$. In addition, knockout of molecules associated with inflammation

This article is protected by copyright. All rights reserved. 
including $\mathrm{CD} 11 \mathrm{c}^{+}$or the overexpression of the anti-inflammatory cytokine IL-10 along with exercise interventions led to protection against high fat diet-induced IR ${ }^{53,57,59}$. Higher muscle macrophage content was inversely correlated with HOMA-IR, GTT, ITT, as well as Akt phosphorylation $^{43,59}$.

The evidence from murine studies was supported by in vitro experiments to investigate the effect of lowering inflammation on glucose uptake and insulin signaling in myotubes. Inflammation neutralization via CD11 $\mathrm{c}^{+}$macrophage ablation ${ }^{57}$ and atherosclerosis-prone apolipoprotein $\mathrm{E}$ (ApoE) knockout ${ }^{60}$ were all shown to improve insulin-stimulated glucose uptake in myotubes, and co-cultures of myotubes with macrophages led to reduced insulin-stimulated glucose uptake $^{56}$. In summary, mice with obesity have insulin resistance, a finding that correlates positively with their muscle macrophage content.

\section{Impact of interventions on murine muscle macrophages and insulin action}

Several studies reported interventions to reduce obesity and inflammation in mice with studies reporting exercise regimens and pharmacotherapies. In the former, a six-week endurance exercise program consisting of 3 days per week of 15 minutes exercise at 10-15 meters/minute led to reduced macrophage F4/80 expression in active mice with obesity versus a control group of sedentary mice with obesity ${ }^{59}$. The second exercise regimen consisted of a 12-week treadmill protocol of 40 minutes per day, 5 days per week at $5 \%$ grade at 12 meters/minute. Exercise reduced the F4/80 and TGF- $\beta 1$ levels associated with $\mathrm{HFD}^{58}$. 
There were several studies examining the use of pharmacotherapy in modulating muscle macrophage content and inflammation with high fat diet-induced obesity. Treatment with the antidiabetic medication Rosiglitazone, a Thiozolinedione, reduced the expression of CD68, CD11c, CCR2, and CCL2 that was increased by HFD. The antidiabetic agent Canagliflozin, a sodium glucose co-transporter 2 inhibitor, decreased mRNA abundance of F4/80 and TNF $\alpha$ in mice with obesity ${ }^{63}$. The use of Quercetin, a plant-based flavonoid, inhibited the rise in gene and protein expression of CCL2 and TNF $\alpha^{54}$. Baricitinib, a Janus kinase (JAK) 1 and 2 inhibitor, reduced T-Lymphocyte but not macrophage markers in skeletal muscle of high fat-fed mice ${ }^{47}$.

\section{Genetic manipulation of murine models and its effects on muscle macrophages}

Several murine models were used to study muscle inflammation in obesity. The overexpression of the anti-inflammatory cytokine IL-10 reduced macrophage levels in skeletal muscle and protected the mice against HFD-related $\mathrm{IR}^{53}$. Ablation of CD11c in macrophages on an HFD resulted in preservation of insulin sensitivity and reduced expression of CD68, TNF $\alpha$, and $\mathrm{CCL}^{57}$.

The blockade of 4-1BB and 4-1BBL, type 2 transmembrane glycoproteins found on T-cells and macrophages and are important in T-Lymphocyte activation, led to reduced obesity-induced skeletal muscle inflammation ${ }^{55}$. In ApoE-/- mice, a mouse model of atherosclerosis with systemic inflammation, Akt phosphorylation was preserved in mice with obesity ${ }^{60}$. One study examined muscle monocyte chemoattractant protein 1 (MCK-MCP1) transgenic mice, over expressing MCP-1(also known as CCL2), which is one of the most important chemokines 
that recruit macrophages to sites of inflammation, and reported dysglycemia and systematic IR with elevated levels of CD68, CD11c, TNF- $\alpha$, IL-1 $\beta$ and Regulated on Activation Normal T-cell Expressed and Secreted (RANTES) compared to wild type mice ${ }^{43}$.

In summary, murine studies inconsistently demonstrated that HFD-induced obesity was associated with an increase in muscle macrophage content and that exercise and pharmacotherapeutic agents lowered macrophage markers in obesity.

\section{Risk of bias across mouse studies}

The risk of bias was assessed using the Systematic Review Center for Laboratory Animal Experimentation (SYRCLE) tool across mouse studies (Table 4) and was unclear or high for multiple study domains.

There was unclear or high risk of bias surrounding sequence generation, allocation concealment and random housing for 17 of the 18 studies. While the blinding of investigators may be difficult due to visible changes in the mice receiving high fat diet, blinding of outcome assessors was reported only in three studies. Random outcome assessment was not documented clearly in the included studies. We rated risk of bias to be unclear for incomplete outcome data because studies did not provide the number of mice randomized to intervention groups, and it was unclear whether all mice were included in the final data analysis. There was high risk of bias surrounding selective outcome reporting in five studies $26,43,52,56,61$, such that data for pre-specified outcomes were not shown or not described in detail mostly when the findings were not significant.

\section{GRADE assessment}


We assessed overall certainty in the evidence using GRADE framework (Table S2) ${ }^{71}$. The assessment found a very low certainty of evidence in the included studies for the outcomes of macrophage content, phenotype, and the association between macrophages and insulin resistance. This was due to ratings of 'serious' to 'very serious' for risk of bias, inconsistency, indirectness, and imprecision. Indirectness was rated as 'serious' due to variability in recruitment, exposure measurement, outcome measurements, and populations. Imprecision was rated as serious due to the small sample sizes. For the mouse studies, we considered inconsistency to be 'not serious' for all outcomes. However, for the human studies, inconsistency was rated as 'serious' for all outcomes due to differences in study populations and measurement techniques for exposure and outcomes. Risk of bias was rated 'serious' among all outcomes for human and murine studies due to issues with participant selection and randomization, confounding bias, and selective reporting.

Due to the variability in macrophage markers and lack of exact results being reported, we were unable to determine the presence of publication bias through funnel plot or Egger's test however we expect that there is publication bias with murine studies.

\section{DISCUSSION}

Skeletal muscle is the largest metabolic organ in humans and mice, and impaired insulin signaling and postprandial glucose disposal in obesity is a critical event that primes the risk for developing T2D, one of the most significant chronic diseases of the $21^{\text {st }}$ century ${ }^{72}$. 
On a mechanistic level, inflammation is one pathway through which obesity can drive muscle insulin resistance and beget T2D. This inflammatory response can result from the local inflammatory cytokines produced by myocytes or immune cells that infiltrate muscle pursuing a chemokine gradient ${ }^{43}$. In addition, the intermyocellular fat depot expands with obesity and attracts monocytes that will contribute to the local inflammatory process. Muscle is also subjected to the influx of systemic inflammatory cytokines and fatty acids that are produced in remote organs including the adipose tissue and arrive to muscle through the circulation ${ }^{73-75}$. The identification of muscle-macrophage crosstalk pathways is critical to unlock strategic approaches to target inflammation and prevent, delay, and treat obesity and T2D. This may significantly improve the health outcomes of billions of people around the world by mitigating the effects of obesity and T2D on health. In this systematic review, we demonstrate that the evidence base for addressing the question of SM and macrophage crosstalk in humans and mice with obesity has limitations.

In human studies, macrophage content and phenotype were variable with obesity in NGT, while in subjects with obesity and IGT, macrophages were either equal or increased. T2D was associated with increased macrophage markers with either enhanced, equal, or reduced antiinflammatory macrophage marker expression. Macrophage content in muscle was positively associated with insulin resistance in NGT and T2D but not in IGT. Rather surprisingly, interventions including exercise and pharmacotherapy did not alter muscle macrophage marker expression in subjects with obesity or T2D. This may be due to short-term nature of all 
interventions, which limits the full understanding of their impact on muscle-immune crosstalk in obesity and diabetes. This is an area that requires further research.

In mice, there was some inconsistency in the reported macrophage expansion in obesity. Most studies reported an increase in total macrophage content and suggested an increase in one marker of anti-inflammatory macrophages/dendritic cells CD11c and increased anti-inflammatory macrophage markers CD206 and CD301b and a reduction in CX3CR1. More consistent evidence of the association of macrophage markers with insulin resistance was reported in mice, and exercise interventions and pharmacotherapy resulted in the reduction of muscle macrophage markers and inflammation.

Reporting of study methodology had limitations among both human and murine studies.

Observational studies in human subjects lacked several details including the reporting of the full profile of obesity and leanness and recruitment strategies. The Strengthening the Reporting of Observational Studies in Epidemiology (STROBE) Statement has been published for improving reporting of observational studies ${ }^{76}$, and endorsement by publishing journals of future studies will likely improve the reporting of eligibility criteria for participants. Use of the STROBE extension for studies in molecular epidemiology (STROBE-ME) can also improve methodological reporting for laboratory-based experimentation among studies investigating biomarkers of inflammation and insulin action in obesity ${ }^{77}$. The majority of murine studies did not report on random sequence generation, allocation concealment, random housing and random outcome assessments. It may be that the methodology 
across included studies was adequate, however it was not possible to judge this based on the data reported, resulting in an unclear risk of bias. Although inadequate reporting of methods may be due to seven mouse studies $26,52,53,56,57,61,62$ being published prior to the availability of the SYRCLE reporting statement for animal studies, a number of recent studies have similar methodological limitations. The Animal Research: Reporting of In Vivo Experiments (ARRIVE) guidelines, published in 2010, aimed to improve the quality of reporting of animal studies ${ }^{78}$. These reporting guidelines are now endorsed by the Enhancing the QUAlity and Transparency Of health Research (EQUATOR) Network, which is an international initiative that aims to increase reliability of published health research by promoting transparency in the reporting of studies ${ }^{79}$. It is critical that investigators use the ARRIVE guidelines to report their experimental work and results to allow the comparisons across studies using their detailed methods. Furthermore, mandated requirements from preclinical journals to use reporting guidelines and submit a completed checklist is important in enhancing the quality of published data and allow replication. This has already been shown to be valuable in clinical research with randomized controlled trials ${ }^{80}$, and can be useful in bench-based research.

\section{Strengths and limitations}

The strengths of this review include the comprehensive literature search and a priori determined approach to investigate macrophage infiltration in SM during obesity in humans and mice to ensure the transparency of the search process. 
Some of the limitations include the variability in reporting of the macrophage content results as relative gene and protein expression, such that we were unable to conduct a meta-analysis. In addition, the differences noted in human and murine muscle macrophage phenotype suggests that murine models are an important tool to study muscle-macrophage crosstalk, however this may not be the case for other immune cells and this requires further assessment. More focus on welldesigned, adequately-powered human and murine studies with well-defined macrophage classification marker sets is critical to provide insights into the muscle immune phenotype in obesity and T2D. This will support the enhancement of the translational potential of murine studies moving forward.

\section{CONCLUSION}

Humans and mice with obesity appear to have a heterogenous muscle-macrophage crosstalk phenotype with certain similarities but some important differences. The adoption of tools including STROBE or STROBE-ME and the recently established ARRIVE guidelines when reporting preclinical and translational research studies will improve transparency and quality of reporting of the research findings. Ultimately, this will enhance the potential for knowledge translation to benefit patients.

\section{Authors' contributions}

MCS is the study guarantor. SR, MB, DP, LB, GRS, MT and MCS were responsible for the development of the study question. SR, MB, GRS, LT, LB, KWW, PWW, RB, DP, MT and MCS were responsible for the study design. SR, LB, MB, PWW, KWW, GRS, LT, DP, RB, and 
MCS developed the search strategy. SR, LB, MB, E.D.S., W.E., PWW, KWW, RB, and MCS executed the search strategy.

SR, PWW, RB, EDS, WE, and MB conducted risk of bias assessments. SR, MB, GRS, KWW, EDS, WE, LT, DP, MT, and MCS were responsible for synthesis and interpretation of data. SR, MB, EDS, WE, and MCS developed the first draft of the manuscript. All authors provided critical revisions of the manuscript and approved the final version. SR \& MB contributed equally to this work.

ACKNOWLEDGEMENTS: We would like to thank Ms. Ajantha Nadarajah, Ms. Ashleen

Kaushal, and Ms. Emily Yang for their help with the editorial details of the manuscript.

Funding: This systematic review received no specific funding. KWW and EDS received funding from the Canada Graduate Scholarship-Masters from the Canadian Institutes of Health Research.

\section{Legends for tables and figures:}

Figure 1. Flow diagram of study inclusion process

Table 1. Summary of human studies

Table 2. Risk of bias summary for human studies

Table 3. Summary of mouse studies

Table 4. Risk of bias summary for mouse studies

Supporting information:

This article is protected by copyright. All rights reserved. 
Table S1. PRISMA Checklist

Table S2. GRADE Table

\section{REFERENCES}

1 Saltiel AR, Olefsky JM. Inflammatory mechanisms linking obesity and metabolic disease. J Clin Invest. 2017; 127: 1-4.

2 Spiegelman BM, Flier JS. Obesity and the regulation of energy balance. Cell. 2001; 104: 531-43.

3 Ezzati M, Riboli E. Behavioral and dietary risk factors for noncommunicable diseases. $N$ Engl J Med. 2013; 369: 954-64.

4 Covic T, Roufeil L, Dziurawiec S. Community beliefs about childhood obesity: its causes, consequences and potential solutions. J Public Health (Oxf). 2007; 29: 123-31.

5 Dehghan M, Akhtar-Danesh N, Merchant AT. Childhood obesity, prevalence and prevention. Nutr J. 2005; 4: 24.

6 Gurnani M, Birken C, Hamilton J. Childhood Obesity: Causes, Consequences, and Management. Pediatr Clin North Am. 2015; 62: 821-40.

7 Sahoo K, Sahoo B, Choudhury AK, Sofi NY, Kumar R, Bhadoria AS. Childhood obesity: causes and consequences. J Family Med Prim Care. 2015; 4: 187-92.

8 Bray GA. Regulation of energy balance: studies on genetic, hypothalamic and dietary obesity. Proc Nutr Soc. 1982; 41: 95-108.

9 Glass CK, Olefsky JM. Inflammation and lipid signaling in the etiology of insulin resistance. Cell Metab. 2012; 15: 635-45.

10 Gregor MF, Hotamisligil GS. Inflammatory mechanisms in obesity. Anпu Rev Immunol. 2011; 29: 415-45.

11 Shulman GI. Cellular mechanisms of insulin resistance. J Clin Invest. 2000; 106: 171-6.

12 Bourlier V, Bouloumie A. Role of macrophage tissue infiltration in obesity and insulin resistance. Diabetes Metab. 2009; 35: 251-60.

13 Lafontan M. Adipose tissue and adipocyte dysregulation. Diabetes Metab. 2014; 40: 1628.

14 Samaan MC. The macrophage at the intersection of immunity and metabolism in obesity. Diabetol Metab Syndr. 2011; 3: 29.

This article is protected by copyright. All rights reserved. 
15 Roszer T. Understanding the Mysterious M2 Macrophage through Activation Markers and Effector Mechanisms. Mediators Inflamm. 2015; 2015: 816460.

16 Nishimura S, Manabe I, Nagasaki M, et al. CD8+ effector T cells contribute to macrophage recruitment and adipose tissue inflammation in obesity. Nat Med. 2009; 15: 914-20.

17 Cancello R, Clement K. Is obesity an inflammatory illness? Role of low -grade inflammation and macrophage infiltration in human white adipose tissue. BJOG. 2006; 113: 1141-47.

18 Gordon S, Pluddemann A, Martinez Estrada F. Macrophage heterogeneity in tissues: phenotypic diversity and functions. Immunol Rev. 2014; 262: 36-55.

19 Gordon S, Pluddemann A. Tissue macrophage heterogeneity: issues and prospects. Semin Immunopathol. 2013; 35: 533-40.

20 Lumeng CN, Bodzin JL, Saltiel AR. Obesity induces a phenotypic switch in adipose tissue macrophage polarization. J Clin Invest. 2007; 117: 175-84.

21 Cancello R, Clement K. Is obesity an inflammatory illness? Role of low -grade inflammation and macrophage infiltration in human white adipose tissue. BJOG. 2006; 113: 1141-47.

22 Cinti S, Mitchell G, Barbatelli G, et al. Adipocyte death defines macrophage localization and function in adipose tissue of obese mice and humans. J Lipid Res. 2005; 46: 2347-55. $23 \mathrm{Xu} \mathrm{H}$, Barnes GT, Yang Q, et al. Chronic inflammation in fat plays a crucial role in the development of obesity-related insulin resistance. J Clin Invest. 2003; 112: 1821-30.

24 Amano SU, Cohen JL, Vangala $\mathrm{P}$, et al. Local proliferation of macrophages contributes to obesity-associated adipose tissue inflammation. Cell Metab. 2014; 19: 162-71.

25 Lumeng CN, DeYoung SM, Bodzin JL, Saltiel AR. Increased inflammatory properties of adipose tissue macrophages recruited during diet-induced obesity. Diabetes. 2007; 56: 16-23.

26 Weisberg SP, McCann D, Desai M, Rosenbaum M, Leibel RL, Ferrante AW Jr. Obesity is associated with macrophage accumulation in adipose tissue. J Clin Invest. 2003; 112: 1796808.

27 Kelley D, Mokan M, Veneman T. Impaired postprandial glucose utilization in noninsulin-dependent diabetes mellitus. Metabolism. 1994; 43: 1549-57.

28 Meyer C, Dostou JM, Welle SL, Gerich JE. Role of human liver, kidney, and skeletal muscle in postprandial glucose homeostasis. Am J Physiol Endocrinol Metab. 2002; 282: E419E27.

29 Baron AD, Laakso M, Brechtel G, Hoit B, Watt C, Edelman SV. Reduced postprandial skeletal muscle blood flow contributes to glucose intolerance in human obesity. J Clin Endocrinol Metab. 1990; 70: 1525-33.

30 Goodpaster BH, Wolf D. Skeletal muscle lipid accumulation in obesity, insulin resistance, and type 2 diabetes. Pediatr Diabetes. 2004; 5: 219-26.

31 Carroll L, Voisey J, Van Daal A. Mouse models of obesity. Clin Dermatol. 2004; 22: 345-49. 
32 Sinasac DS, Riordan JD, Spiezio SH, Yandell BS, Croniger CM, Nadeau JH. Genetic control of obesity, glucose homeostasis, dyslipidemia and fatty liver in a mouse model of dietinduced metabolic syndrome. Int J Obes (Lond). 2016; 40: 346-55.

33 Hileman SM, Pierroz DD, Masuzaki H, et al. Characterizaton of short isoforms of the leptin receptor in rat cerebral microvessels and of brain uptake of leptin in mouse models of obesity. Endocrinology. 2002; 143: 775-83.

34 Nawrocki AR, Rajala MW, Tomas E, et al. Mice lacking adiponectin show decreased hepatic insulin sensitivity and reduced responsiveness to peroxisome proliferator-activated receptor $\gamma$ agonists. J Biol Chem. 2006; 281: 2654-60.

35 Uysal KT, Wiesbrock SM, Marino MW, Hotamisligil GS. Protection from obesityinduced insulin resistance in mice lacking TNF- $\alpha$ function. Nature. 1997; 389: 610-4.

$36 \mathrm{Xu}$ J, Lloyd DJ, Hale C, et al. Fibroblast growth factor 21 reverses hepatic steatosis, increases energy expenditure, and improves insulin sensitivity in diet-induced obese mice. Diabetes. 2009; 58: 250-59.

37 Shay T, Jojic V, Zuk O, et al. Conservation and divergence in the transcriptional programs of the human and mouse immune systems. Proc Natl Acad Sci U S A. 2013; 110: 294651 .

38 Bhatt M, Rudrapatna S, Banfield L, et al. Evaluating the evidence for macrophage presence in skeletal muscle and its relation to insulin resistance in obese mice and humans: a systematic review protocol. BMC Res Notes. 2017; 10: 374.

39 Wells GA, Shea B, O'Connell D, et al. The Newcastle-Ottawa Scale (NOS) for assessing the quality of nonrandomised studies in meta-analyses. http://www.ohri.ca/programs/clinical_epidemiology/oxford.asp Accessed July 26, 2017. 40 Hooijmans CR, Rovers MM, de Vries RB, Leenaars M, Ritskes-Hoitinga M, Langendam MW. SYRCLE's risk of bias tool for animal studies. BMC Med Res Methodol. 2014; 14: 43.

41 Varma V, Yao-Borengasser A, Rasouli N, et al. Muscle inflammatory response and insulin resistance: synergistic interaction between macrophages and fatty acids leads to impaired insulin action. Am J Physiol Endocrinol Metab. 2009; 296: E1300-10.

42 Fink LN, Costford SR, Lee YS, et al. Pro-inflammatory macrophages increase in skeletal muscle of high fat-fed mice and correlate with metabolic risk markers in humans. Obesity (Silver Spring). 2014; 22: 747-57.

43 Patsouris D, Cao JJ, Vial G, et al. Insulin resistance is associated with MCP1-mediated macrophage accumulation in skeletal muscle in mice and humans. PLoS One. 2014; 9: e110653. 44 Fink LN, Oberbach A, Costford SR, et al. Expression of anti-inflammatory macrophage genes within skeletal muscle correlates with insulin sensitivity in human obesity and type 2 diabetes. Diabetologia. 2013; 56: 1623-8.

45 Liu D, Morales FE, IglayReger HB, et al. Expression of macrophage genes within skeletal muscle correlates inversely with adiposity and insulin resistance in humans. Appl Physiol Nutr Metab. 2017; 43: 187-193. 
46 Tam CS, Sparks LM, Johannsen DL, Covington JD, Church TS, Ravussin E. Low macrophage accumulation in skeletal muscle of obese type 2 diabetics and elderly subjects. Obesity (Silver Spring). 2012; 20: 1530-3.

47 Khan IM, Perrard XY, Brunner G, et al. Intermuscular and perimuscular fat expansion in obesity correlates with skeletal muscle $\mathrm{T}$ cell and macrophage infiltration and insulin resistance. Int J Obes (Lond). 2015; 39: 1607-18.

48 Bruun JM, Helge JW, Richelsen B, Stallknecht B. Diet and exercise reduce low-grade inflammation and macrophage infiltration in adipose tissue but not in skeletal muscle in severely obese subjects. Am J Physiol Endocrinol Metab. 2006; 290: E961-7.

49 Di Gregorio GB, Yao-Borengasser A, Rasouli N, et al. Expression of CD68 and macrophage chemoattractant protein-1 genes in human adipose and muscle tissues: association with cytokine expression, insulin resistance, and reduction by pioglitazone. Diabetes. 2005; 54: 2305-13.

50 Kim TH, Choi SE, Ha ES, et al. IL-6 induction of TLR-4 gene expression via STAT3 has an effect on insulin resistance in human skeletal muscle. Acta Diabetol. 2013; 50: 189-200.

51 Enos RT, Velazquez KT, Murphy EA. Insight into the impact of dietary saturated fat on tissue-specific cellular processes underlying obesity-related diseases. J Nutr Biochem. 2014; 25: 600-12.

52 Hevener AL, Olefsky JM, Reichart D, et al. Macrophage PPAR gamma is required for normal skeletal muscle and hepatic insulin sensitivity and full antidiabetic effects of thiazolidinediones. J Clin Invest. 2007; 117: 1658-69.

53 Hong EG, Ko HJ, Cho YR, et al. Interleukin-10 prevents diet-induced insulin resistance by attenuating macrophage and cytokine response in skeletal muscle. Diabetes. 2009; 58: 252535 .

54 Le NH, Kim CS, Park T, et al. Quercetin protects against obesity-induced skeletal muscle inflammation and atrophy. Mediators Inflamm. 2014; 2014: 834294.

55 Le NH, Kim CS, Tu TH, et al. Blockade of 4-1BB and 4-1BBL interaction reduces obesity-induced skeletal muscle inflammation. Mediators Inflamm. 2013; 2013: 865159.

56 Nguyen MT, Favelyukis S, Nguyen AK, et al. A subpopulation of macrophages infiltrates hypertrophic adipose tissue and is activated by free fatty acids via Toll-like receptors 2 and 4 and JNK-dependent pathways. J Biol Chem. 2007; 282: 35279-92.

57 Patsouris D, Li PP, Thapar D, Chapman J, Olefsky JM, Neels JG. Ablation of CD11cpositive cells normalizes insulin sensitivity in obese insulin resistant animals. Cell Metab. 2008; 8: 301-9.

58 Pincu Y, Linden MA, Zou K, Baynard T, Boppart MD. The effects of high fat diet and moderate exercise on TGFbeta1 and collagen deposition in mouse skeletal muscle. Cytokine. 2015; 73: 23-9.

59 Samaan MC, Marcinko K, Sikkema S, et al. Endurance interval training in obese mice reduces muscle inflammation and macrophage content independently of weight loss. Physiol Rep. 2014; 2. 
60 Wang J, Perrard XD, Perrard JL, et al. ApoE and the role of very low density lipoproteins in adipose tissue inflammation. Atherosclerosis. 2012; 223: 342-9.

$61 \mathrm{Xu} \mathrm{H}$, Barnes GT, Yang Q, et al. Chronic inflammation in fat plays a crucial role in the development of obesity-related insulin resistance. J Clin Invest. 2003; 112: 1821-30.

62 Guri AJ. Abscisic acid ameliorates glucose tolerance and obesity-induced inflammation [dissertation]. Blacksburg: Virginia Polytechnic Institute and State University; 2007.

63 Naznin F, Sakoda H, Okada T, et al. Canagliflozin, a sodium glucose cotransporter 2 inhibitor, attenuates obesity-induced inflammation in the nodose ganglion, hypothalamus, and skeletal muscle of mice. Eur J Pharmacol. 2017; 794: 37-44.

$64 \quad$ Nwadozi E, Roudier E, Rullman E, et al. Endothelial FoxO proteins impair insulin sensitivity and restrain muscle angiogenesis in response to a high-fat diet. FASEB J.2016; 30: 3039-52.

65 Shin HY, Kang HT. Recent trends in the prevalence of underweight, overweight, and obesity in Korean adults: The Korean National Health and Nutrition Examination Survey from 1998 to 2014. J Epidemiol. 2017; 27: 413-19.

66 Holness CL, Simmons DL. Molecular cloning of CD68, a human macrophage marker related to lysosomal glycoproteins. Blood. 1993; 81: 1607-13.

67 Wang J, Pan Y, Hong Y, Zhang Q-Y, Wang X-N, Kong L-D. Quercetin Protects against Cadmium-Induced Renal Uric Acid Transport System Alteration and Lipid Metabolism Disorder in Rats. Evid Based Complement Alternat Med. 2012; 2012: 548430.

68 Guri AJ, Hontecillas R, Bassaganya-Riera J. Obesity promotes macrophage infiltration into both adipose tissue and skeletal muscle and impairs the regulatory $C D 4+T$ cell compartment [dissertation]. Blacksburg: Virginia Polytechnic Institute and State University; 2007.

69 Wang XA, Zhang R, Zhang S, et al. Interferon regulatory factor 7 deficiency prevents diet-induced obesity and insulin resistance. Am J Physiol Endocrinol Metab. 2013; 305: E48595.

70 Patsouris D, Cao JJ, Vial G, et al. Insulin resistance is associated with MCP1-mediated macrophage accumulation in skeletal muscle in mice and humans. PLoS One. 2014; 9: e110653 71 Guyatt GH, Oxman AD, Kunz R, et al. GRADE guidelines: 7. Rating the quality of evidence--inconsistency. J Clin Epidemiol. 2011; 64: 1294-302.

72 Zimmet PZ, Magliano DJ, Herman WH, Shaw JE, Diabetes: a 21st century challenge. Lancet Diabetes Endocrinol. 2014; 2: 56-64.

73 Samaan MC. The macrophage at the intersection of immunity and metabolism in obesity. Diabetol Metab Syndr. 2011; 3: 29.

74 Lauterbach MA, Wunderlich FT. Macrophage function in obesity-induced inflammation and insulin resistance. Pflügers Arch. 2017; 469: 385-96.

75 Murray PJ, Wynn TA. Protective and pathogenic functions of macrophage subsets. Nat Rev Immunol. 2011; 11: 723-37. 
76 Von Elm E, Altman DG, Egger M, et al. The Strengthening the Reporting of Observational Studies in Epidemiology (STROBE) statement: guidelines for reporting observational studies. Prev Med. 2007; 45: 247-51.

77 Gallo V, Egger M, McCormack V, et al. STrengthening the Reporting of OBservational studies in Epidemiology-Molecular Epidemiology (STROBE-ME): an extension of the STROBE Statement. Mutagenesis. 2011; 27: 17-29.

78 Kilkenny C, Browne WJ, Cuthill IC, Emerson M, Altman DG. Improving bioscience research reporting: the ARRIVE guidelines for reporting animal research. PLoS Biol. 2010; 8: e1000412.

79 Altman DG, Simera I, Hoey J, Moher D, Schulz K. EQUATOR: reporting guidelines for health research. Lancet. 2008; 371: 1149-50.

80 Moher D, Jones A, Lepage L, CONSORT Group. Use of the CONSORT statement and quality of reports of randomized trials: a comparative before-and-after evaluation. JAMA. 2001; 285: 1992-95.

This article is protected by copyright. All rights reserved. 


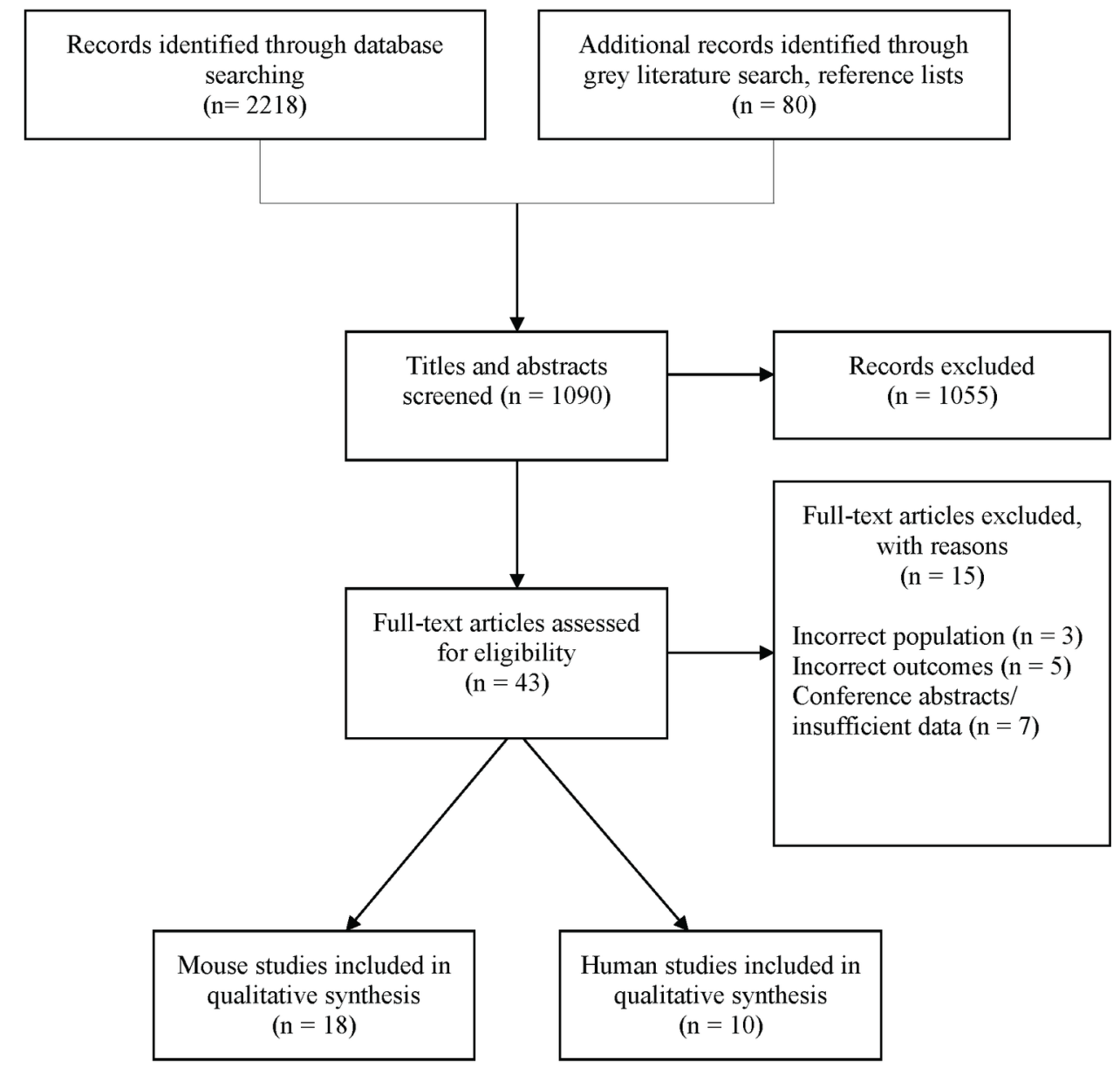

Figure 1. Flow diagram of study inclusion process

OBR_12922_f1.TIF

This article is protected by copyright. All rights reserved. 


\section{University Library}

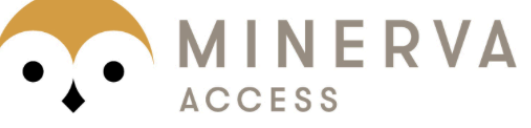

A gateway to Melbourne's research publications

Minerva Access is the Institutional Repository of The University of Melbourne

Author/s:

Rudrapatna, S;Bhatt, M;Wang, K-W;Bierbrier, R;Wang, P-W;Banfield, L;Elsheikh, W;Sims, ED;Peterson, D;Thabane, L;Tarnopolsky, MA;Steinberg, GR;Samaan, MC

Title:

Obesity and muscle-macrophage crosstalk in humans and mice: A systematic review.

Date:

2019-11

Citation:

Rudrapatna, S., Bhatt, M., Wang, K. -W., Bierbrier, R., Wang, P. -W., Banfield, L., Elsheikh, W., Sims, E. D., Peterson, D., Thabane, L., Tarnopolsky, M. A., Steinberg, G. R. \& Samaan, M. C. (2019). Obesity and muscle-macrophage crosstalk in humans and mice: A systematic review.. Obes Rev, 20 (11), pp.1572-1596. https://doi.org/10.1111/obr.12922.

Persistent Link:

http://hdl.handle.net/11343/286297 